\title{
Osteoblastoma of the mandible: a case report with immunohistochemical evaluation
}

\author{
Evanthia Chrysomali ${ }^{1}$, Ourania Schoinohoriti ${ }^{2}$, Nadia Theologie-Lygidakis ${ }^{2}$, \\ Lambros Goutzanis ${ }^{2}$, Ioannis Iatrou ${ }^{2}$ \\ ${ }^{1}$ Department of Oral Pathology and Medicine, School of Dentistry, University of Athens, Athens, Greece; \\ ${ }^{2}$ Department of Oral and Maxillofacial Surgery, Children’s Hospital "P. and A. Kyriakou”, School of Dentistry, University of Athens, \\ Athens, Greece. \\ Email: our_schoinohoriti@yahoo.com
}

Received 19 September 2011; revised 23 October 2011; accepted 5 November 2011.

\begin{abstract}
Background: Osteoblastoma, a rare osteoblastic tumor, constitutes approximately $1 \%$ of all primary bone tumors. Aim: The purpose of this report is to present an osteoblastoma of the mandible, with particular emphasis on the differential diagnosis of this rare tumor. Methods-Results: the lesion showed osteoblastlike cells, stromal cells, osteoclast-like cells and interlacing trabeculae of osteoid. Because of the high cellularity of the tumor, immunohistochemical analysis was performed using the cell proliferation marker $\mathrm{Ki}-67$, the p53 and the anti-apoptotic protein Bcl-2. The lesion demonstrated low to moderate proliferative activity and no immunoreactivity was detected for $p 53$. An interesting finding was the Bcl-2 expression by the multinucleated osteoclast-like giant cells, in contrast to the lack of Bcl-2 expression from osteoblast-like and stromal tumor cells. Conclusions: To our knowledge the Bcl-2 expression has not yet been investigated in benign or malignant osteoblastic tumors. This finding may be related to the molecular mechanisms regulating the apoptosis of osteoclast-like tumor cells or their function.
\end{abstract}

Keywords: Osteoblastoma; Mandible; Reconstruction; Immunohistochemistry; Ki-67; Bcl-2; p53; Bone Tumors

\section{INTRODUCTION}

Osteoblastoma represents about $1 \%$ of all primary bone tumors. Approximately $11 \%$ of the bone osteoblastomas are localized to the skull and nearly half of these cases involve the mandilble [1,2]. Osteoblastoma usually appears in the second decade of life as a painful expansion of the bone cortex with non specific radiographic appearance. Histologically, it is characterized by osteoblast- like cells proliferation forming trabeculae of osteoid and immature bone in a highly vascularized stroma [2-6] These cells are usually uniform in the benign or "classical” osteoblastoma, but they may exhibit nuclear hyperchromatism or/and pleomorphism with features of epithelioid appearance in the "atypical type" of osteoblastoma $[5,7]$.

The most important diagnostic consideration is to differentiate osteoblastoma from osteoblastic osteosarcoma, especially in case of a borderline osteoblastic lesion intermediate osteoblastoma and osteosarcoma [2,4,5,7]. In the maxillofacial region the diagnosis of osteoblastoma is often difficult and careful clinicopathological correlation may be needed, since many jaw lesions may present similar overlapping demographic, clinical, radiographic and histopathological features $[3,4]$.

The purpose of this article is to present a case of osteoblastoma involving the anterior mandible with particular emphasis on the differential diagnosis and the immunohistochemical features of this rare tumor using the cell proliferation marker Ki67, the p53 and the antiapoptotic protein Bcl-2.

\section{CASE REPORT}

A 14-year-old female with free medical history was referred to our clinic because of a 6-month history of progressive painful discomfort located to the anterior mandible. Oral examination showed a minimal expansion of the labial cortex at the anterior parasymphysal area of the mandible related to the incisors, canines and right first premolar. Motility of the adjacent teeth was noted, but the teeth were vital. There was no paraesthesia, the overlying mucosa was intact and no regional lymph nodes were palpable. Orthopantomogram revealed an irregularly-shaped radiolucency without sclerotic borders, in the anterior mandible extending from the right first premolar to the left canine and from the alveolar 
crest to the body of the mandible producing root displacement, but not resorption (Figure 1(a)). Computed tomography scan (Figure 1(b)) showed a radiodense lesion with a peripheral rim of calcification perforating both labial and lingual cortices centrally and expanding the labial cortex peripherally.

The histopathological examination, after an incisional biopsy, showed benign osteoblastic tumor consistent with osteoblastoma. An en bloc resection of the tumor was decided under general anaesthesia. The gross biopsy specimen consisted of a relatively compact mass of reddish to brown friable tumor tissue with irregular surface, resected with a macroscopically healthy block of mandibular bone, measuring $4 \times 2 \times 2.5 \mathrm{~cm}$. After lavish irrigation the osseous defect was reconstructed with a bone graft (a block of cortical bone measuring $2 \times 2.5 \times 0.5$ $\mathrm{cm}$ along with chips of cancellous bone) harvested from the anterior iliac crest at the same surgical time.

Histologically, the lesion showed relatively high cellularity consisting of osteoblast-like cells, stromal cells, osteoclast-like cells, immature bone and osteoid deposition. The interlacing, irregularly-shaped bone trabeculae showed variable thickness and maturation and rimmed by a line of osteoblast-like polygonal cells with abundant eosinophilic cytoplasm and round to oval nuclei (Figure 2(a)). Multinucleated osteoclast-like giant cells were
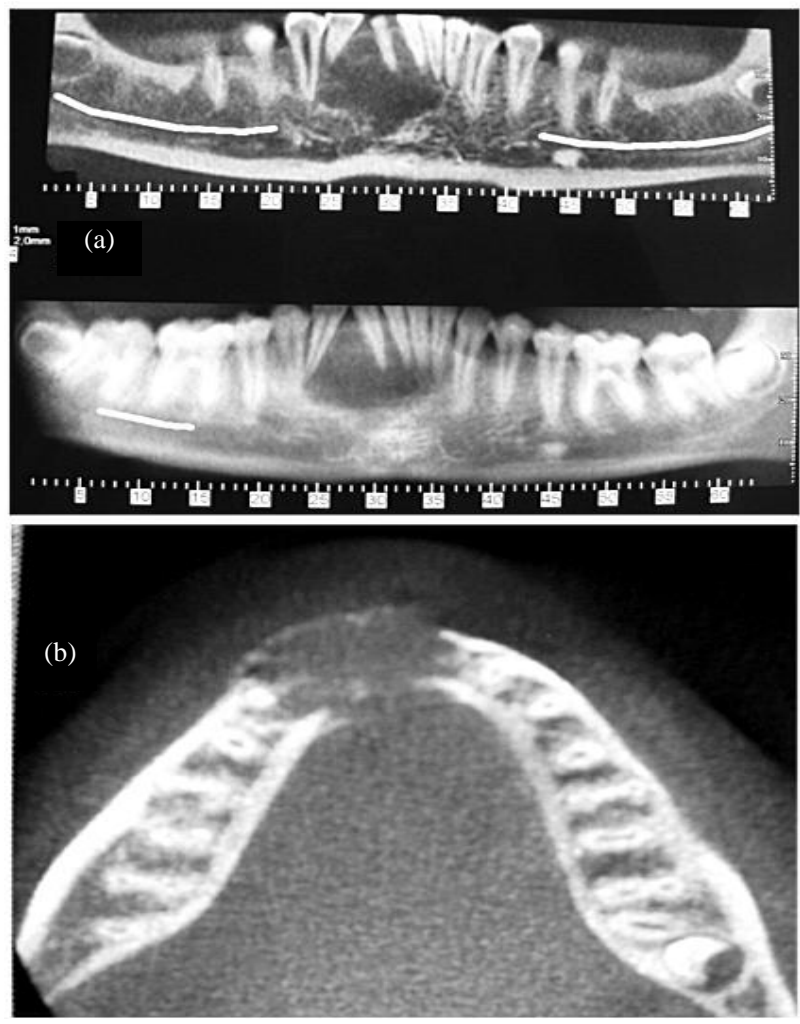

Figure 1. (a) Preoperative orthopantomogram (a) and (b) preoperative CT scan. dispersed through the tumor among spindle shaped stromal cells (Figure 2(b)). Numerous large dilated capillaries, as well as focal hemorrhage were seen in the inter-trabecular stroma of loose fibrovascular tissue. Epithelioid osteoblasts, cytologic atypia, or pleomorphism were not observed. There was no evidence of infiltrated residual bone suggestive of permeative growth pattern. The final diagnosis was osteoblastoma excised upon healthy margins. Because of the highly cellular histopathologic appearance and the occasional, but not abnormal, mitotic activity, immunohistochemical analysis was performed using a strepta-avidin-biotin system and monoclonal antibodies to Ki-67 antigen (clone MIB-1, Dako), p53 protein (clone DO7, Dako) and Bcl-2 protein (clone 124, Dako, Glostrup, Denmark). A strong Bcl-2 protein immunoreactivity was detected in the cytoplasm of multinucleated osteoclast-like cells but limited, if any, reactivity in the stromal and osteoblast-like cells (Figure 2(c)). A weak expression for Ki-67 was detectable in the nuclei of osteoblast-like and stromal cells, in a percentage ranging from $10 \%$ to $25 \%$ of the tumor cells (Figure 2(d)), while no immunoreactivity was detected for p53.

The patient recovered well; she was asymptomatic without any signs of recurrence on follow-up evaluation after one year. After the micro-plate was removed, an orthopantomogram revealed the excellent osseous integration of the graft (Figure 3). A CT scan of the patient 2 years postoperatively documented the lack of recurrence at the operative site (Figure 4).

\section{DISCUSSION}

The differential diagnosis of osteoblastoma includes many jawbone lesions. This case was differentiated from osteoid osteoma due to the lack of the characteristic central bone nidus, which is a feature seen in osteoid osteoma $[3,4,8]$. The clinical findings, such as the large tumor size (over $2 \mathrm{~cm}$ ) and the symptoms (pain, local tenderness/ discomfort) are features usually associated with osteoblastoma [2-4]. Because of the histopathological similarity of osteoblastoma with cementoblastoma, the distinction between them is difficult, but the unique characteristic feature of the latter is its fusion to the root of the adjacent tooth [4]. The tumor was closely related to the adjacent teeth displacing the roots in our case, but it was not fused to them excluding the diagnosis of cementoblastoma.

The term "aggressive osteoblastoma" has been considered appropriate for a subset of lesions which display locally aggressive growth pattern and have atypical histopathological features [5,7]. Aggressive or atypical osteoblastoma is distinguished from classical osteoblastoma due to the presence of large epithelioid, bizarre looking osteoblasts, more osteoclast-like giant cells and 


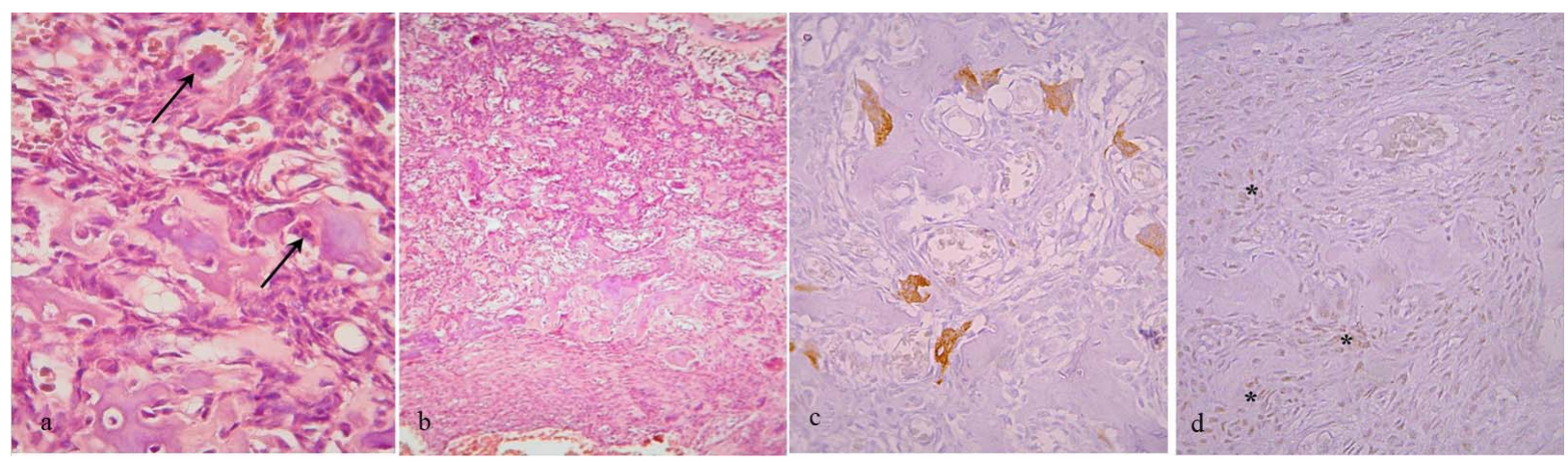

Figure 2. (a) Trabeculae rimmed by polygonal cells resembling osteoblasts; few sparse multinucleated osteoclast-like giant cells (arrows) are noted (HXE, original magnification $\times 120$ ); (b) Irregularly shaped and variably thick bone trabeculae and osteoid deposition (HXE, original magnification $\times 25$ ); (c) Positivity of the multinucleated osteoclast-like giant cells and negativity of the osteoblast-like cells for bcl-2 immunostaining; (d) Weak positivity of stromal cells (marked with asterisks) for Ki67 immunostaining.

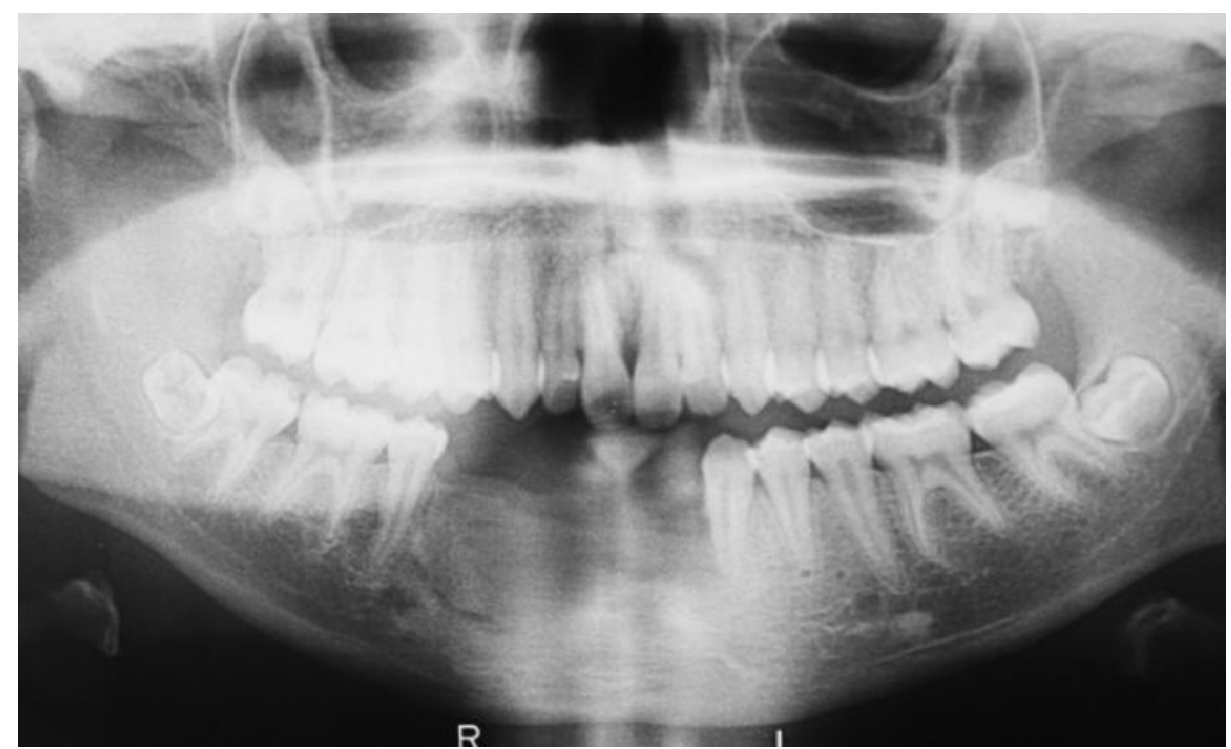

Figure 3. Orthopantomogram of the patient after micro-plate removal.

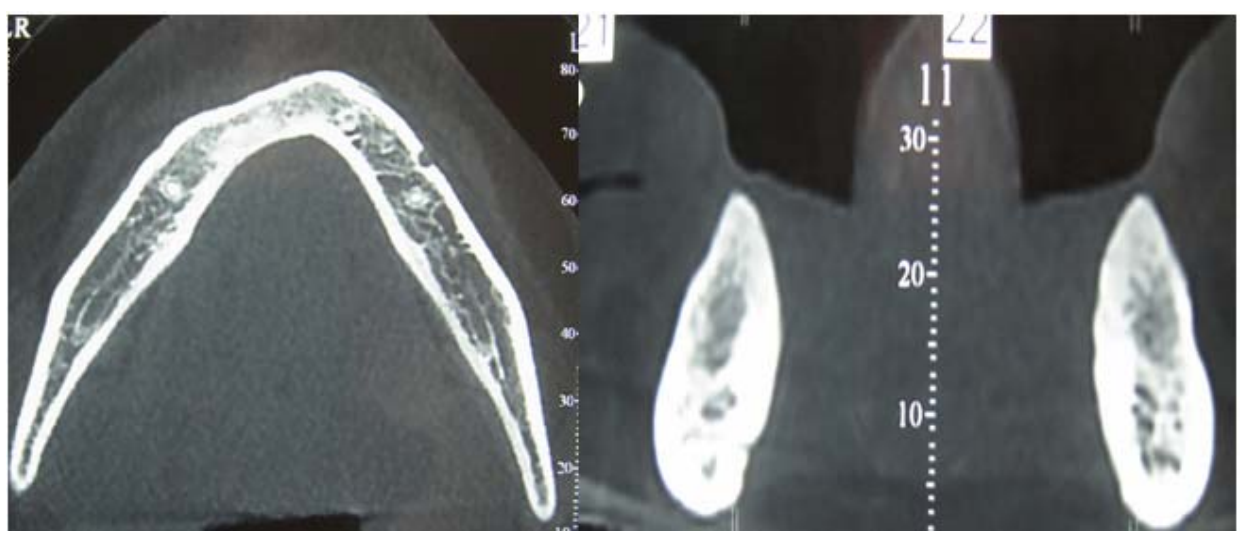

Figure 4. CT scan of the patient 2 years postoperatively.

more abundant atypical osteoid. Because of the variable histological appearance of osteoblastoma, differentiation from osteosarcoma may occasionally be challenging, but the presence of dysplastic features, fine compacted lace- 
like osteoid strands, invasion into host bone, high rate of mitotic activity or atypical mitoses aid in the diagnosis of osteosarcoma [1,2,4,5].

Despite the high cellularity in the case presented here, there was no evidence of epithelioid or dysplastic features, or growth pattern suggestive of malignancy. The Ki-67 immunostaining disclosed a relatively low to moderate cell proliferation index and the tumor cells did not show any immunoreactivity for the p53 protein. Oliveira et al. investigated the cell proliferation marker PCNA, as well as the p53 immunohistochemical expression and p53 gene mutations in classical, atypical osteoblastomas in comparison to osteosarcomas [7]. Atypical osteoblastomas, osteosarcomas and tumor recurrence were statistically correlated with a high PCNA labelling index and p53 immunoexpression [7].

The histopathological and immunohistochemical findings in our case were indicative for the classical osteoblastoma. A long follow-up period may be needed to draw firm conclusions concerning the benign or potential aggressive clinical course of this lesion. Aggressive behavior may be within the biologic spectrum of osteoblastomas; the histopathology may not appear to be a reliable predictor of aggressiveness [1]. The predictive value of cell proliferation or other molecular markers in the biologic behavior of osteoblastoma variants remains to be completely determined.

The Bcl-2 gene and its protein product promote cellular survival; therefore correlation of the $\mathrm{Bcl}-2$ expression with clinical outcome has been examined in many types of tumors. Recently the Bcl-2 protein expression has been correlated-along with other biomarkers - with the progression and prognosis of osteosarcoma [9]; however its expression has not yet, to our knowledge, been investigated in benign osteoblastic tumors. An interesting finding in the present case was the immunohistochemical detection of the Bcl-2 protein in the multinucleated osteoclast-like giant cells, in contrast to the lack of expression of this protein in the osteoblast-like and stromal tumor cells. This finding may be related to the molecular mechanisms regulating the apoptosis of osteoclast-like tumor cells or their function. Anti-apoptotic Bcl-2 family members such as Bcl-2 and Bcl-xL seem to play a significant role, not only in the apoptosis of osteoclasts, but also in the bone resorbing function of these cells [10].

The treatment options of osteoblastoma include conservative surgical excision, excision with vigorous $\mathrm{Cu}-$ rettage followed by bur ablation of the margins and copious irritation or en block resection [4]. Local recurrence related to inadequately removed tumors have been reported in a rate of $14 \%$ [11]. Lesions treated with en bloc resection or resection yielding tumor-free margins exhibit minimal likelihood of recurrence; we believe that such radical procedures are fully justified in pediatric patients suffering from potentially aggressive lesions like osteoblastomas. Despite the propensity of recurrence, primary reconstruction seems to be worthwhile, and should be taken into account when mandibular integrity can be preserved. Long-term follow-up is recommended, of at least 2 years, not only to minimize the risk of undiagnosed recurrence, but also to guarantee the grafts' survival and integration.

\section{REFERENCES}

[1] Lucas, D., Unni, K., McLeod, R., O’Connor, M. and Sim, F. (1994) Osteoblastoma: Clinicopathologic study of 306 cases. Human Pathology, 25, 117-134. doi:10.1016/0046-8177(94)90267-4

[2] Capodiferro, S., Maiorano, E., Giardina, C., Lacaita, M., Lo Muzio, L. and Favia, G. (2005) Osteoblastoma of the mandible: Clinicopathologic study of four cases and literature review. Head and Neck, 27, 616-621. doi:10.1002/hed.20192

[3] Jones, A., Prihoda, T., Kacher, J., Odingo, N. and Freedman, P. (2006) Osteoblastoma of the maxilla and mandible: A report of 24 cases, review of the literature and discussion of its relationship to osteoid osteoma of the jaws. Oral Surgery, Oral Medicine, Oral Pathology, Oral Radiology \& Endodontics, 102, 639-650. doi:10.1016/j.tripleo.2005.09.004

[4] Rawal, Y., Angiero, F., Allen, C., Kalmar, J., Sedghizadeh, P. and Steinhilber, A. (2006) Gnathic osteoblastoma: Clinicopathologic review of seven cases with long-term follow-up. Oral Oncology, 42, 123-130. doi:10.1016/j.oraloncology.2005.04.016v

[5] Lypka, M., Goos, R., Yamashita, D. and Melrose, R. (2008) Aggressive osteoblastoma of the mandible. International Journal of Oral and Maxillofacial Surgery, 37, 675-678. doi:10.1016/j.ijom.2008.01.013

[6] Alvares, C.A., Gião, D.M., Casati, A.L., Negrão, F.R. and Sant'Ana, E. (2005) Osteoblastoma of the mandible: Systematic review of the literature and report of a case. Dentomaxillofacial Radiology, 34, 1-8. doi:10.1259/dmfr/24385194

[7] Oliveira De, C., Mendonça, B., Camargo De, O., Pinto, E., Nascimento, S., et al. (2007) Classical osteoblastoma, atypical osteoblastoma, and osteosarcoma. A comparative study based on clinical, histological, and biological parameters. Clinics, 62, 167-174. doi:10.1590/S1807-59322007000200012

[8] Van der Waal, L., Greebe, R. and Elias, E. (1983) Benign osteoblastoma or osteoid osteoma of the maxilla. International Journal of Oral and Maxillofacial Surgery, 12, 355-358.

[9] Wu, X., Cai, Z.D., Lou, L.M. and Zhu, Y.B. (2011) Expressions of p53, c-MYC, BCL-2 and apoptotic index in human osteosarcoma and their correlations with prognosis of patients. Cancer Epidemiology, Epub Ahead of Print.

[10] Iwasawa, M., Miyazaki, T., Nagase, Y., Akiyama, T., Kadono, Y., Nakamura, M. et al. (2009) The antiapop- 
totic protein $\mathrm{Bcl}-\mathrm{xL}$ negatively regulates the bone resorbing activity of osteoclasts in mice. Journal of Clinical Investigation, 119, 3149-3159.

[11] Gordon, S., MacIntosh, R. and Wesley, R. (2001) A review of osteoblastoma and case report of metachronous osteoblastoma and unicystic ameloblastoma. Oral Surgery, Oral Medicine, Oral Pathology, Oral Radiology \& Endodontics, 91, 570-575.

doi:10.1067/moe.2001.113349 